\title{
INT-MANUS: Interactive Production Control in a Distributed Environment
}

\author{
T. Schlegel ${ }^{1}$, A. Srinivasan ${ }^{1}$, M. Foursa ${ }^{2}$, M. Bogen ${ }^{2}$, R. Narayanan ${ }^{2}$, D. d'Angelo², \\ G. Haidegger ${ }^{3}$, I. Mezgar ${ }^{3}$, J. Canou 4 , D. Sallé4, F. Meo ${ }^{5}$, J. Agirre Ibarbia ${ }^{6}$, \\ A. Herrmann Praturlon ${ }^{7}$ \\ ${ }^{1}$ Fraunhofer IAO, Nobelstr. 12, 70569 Stuttgart, Germany, \\ ${ }^{2}$ Fraunhofer IAIS, ${ }^{3} \mathrm{CIM}$-EXP, ${ }^{4}$ Robosoft, ${ }^{5}$ FIDIA, ${ }^{6}$ FATRONIK, ${ }^{7}$ Centro Ricerche Fiat \\ ${ }^{1}\{$ Thomas.Schlegel, Aravind.Srinivasan\}@iao.fraunhofer.de, \\ ${ }^{2}\{$ Maxim.Foursa, Manfred.Bogen, Rejin.Narayanan, David.D-Angelo\}@iais.fraunhofer.de, \\ ${ }^{3}\{$ Haidegger, Mezgar\}@sztaki.hu, \\ ${ }_{4}^{4}$ Joseph.Canou, Damien.Salle $\} @$ robosoft.fr, \\ 5F.Meo@fidia.it, ${ }^{6}$ JAgirre@fatronik.com, ${ }^{7}$ Anja.HerrmannPraturlon@crf.it
}

\begin{abstract}
The European research project INT-MANUS embedded in the I*PROMS European network of excellence addresses the increasing demand for flexibility and adaptivity, which is summarized by rapid reconfigurations of complete factories as well as related aspects in Human Computer Interaction (HCI), Software, and Production Systems. The project's main goal is to develop a new technology for the production plants of the future, the Smart Connected Control Platform (SCCP). This platform allows controlling a factory with the help of an open distributed learning agent platform that integrates machines, robots, and human personnel.
\end{abstract}

Keywords: Production Service Bus (PSB), Smart-Connected-Control Platform (SCCP), Mobile Control System, real-time, Advanced Maintenance, Augmented Reality, Virtual Reality, Knowledge Repository, tracking system.

Today's production and manufacturing systems are challenged by an increasing demand for flexibility and adaptivity which is summarized by the rapid reconfiguration of a factory. While improvements are already being undertaken with respect to technical requirements, interactive processes and interaction technology in a highly dynamic environment still needs further attention. The European research project INT-MANUS [1] has been launched from the I*PROMS European network of excellence [2] in order to address these issues in addition to aspects of Human Computer Interface (HCI), Software, and Production Systems.

The Smart-Connected-Control Platform (SCCP) for manufacturing enterprises allows controlling a production plant with help of an open distributed learning agent platform that integrates machines, robots, and human personnel. The Smart Connected Control Platform will allow higher automation capabilities in production, delivering a decentralized concept for connecting machines and interaction devices. Ubiquitous augmented reality technology will provide better control abilities for factories, starting with an enhanced communication infrastructure. Integrating 
2 T. Schlegel, A. Srinivasan, M. Foursa, M. Bogen, R. Narayanan, D. d'Angelo,

G. Haidegger, I. Mezgar, J. Canou, D. Sallé, F. Meo, J. Agirre Ibarbia, A. Herrmann Praturlon

interfaces and support for human personnel in every aspect of the production process thereby leads to a better control of the production and manufacturing process

\section{Interfaces}

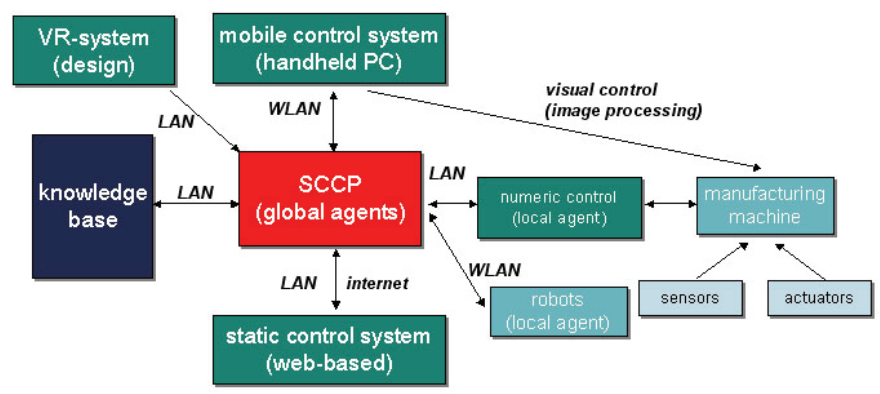

Fig. 1. Overall System Architecture

Each peer (machine tool, robot or other device) is equipped with a local control system that is in charge of local control and communication with the Enterprise Service Bus (ESB). Data collected from sensors are partially processed by the local control systems and after filtering sent over ESB to SCCP services for storing and higher-level analysis. The system then applies its decision-support techniques and if necessary sends updates to control systems and notifications.

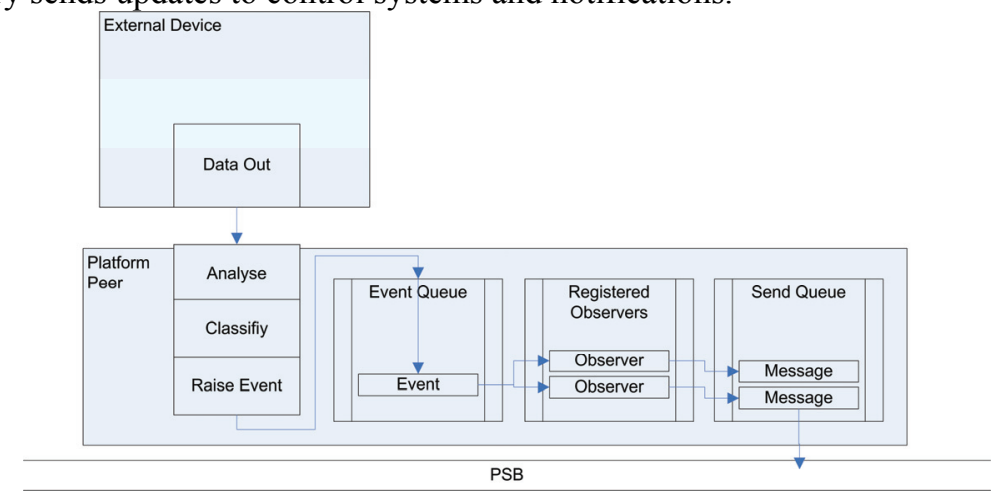

Fig. 2. Schematic Workflow Platform Peer

Connecting numerous devices to the SCCP communication infrastructure, a common access platform is needed. This component, the platform peer, fulfils an important task. It receives (e.g. sensor) data from the device attached and makes them accessible to other peers. Therefore the data have to be collected, classified and referenced to the semantic data description model, a part of the model ontology available in the network. Two processes for the distribution of semantic referenced/annotated data are designated. The Platform Peer can proactively send the data (including a classification reference) to a peer that is known to need them. The more recommended process is having the Platform Peer providing events for each data that can be observed by interested peers. This way the Platform Peer does not 


\section{INT-MANUS: Interactive Production Control}

in a Distributed Environment 3

need explicit knowledge of workflow and data routing. Thus the semantic backbone will mainly use the event based data transmission.

To provide maximum flexibility it is reasonable to collect all occurring events in a single event queue. Events can originate from different sources: From the platform peers view, three inputs can be differentiated: Calls reaching the PSBService interface, user interaction via the connected web interface and events from a potentially connected machine. A fourth event origin is the internal Peer Infrastructure Manager, which is responsible for the generation of periodically upcoming Checks (e.g. network split etc.). The peer control is responsible to map raising events to matching actions. Actions can operate on available status information from the Reactive Interface (machine of/of etc.) and on the three output modules: PSB Client, Web Interface and Machine Control Module. Actions for some occurring events may not be available at the local peer. Then the platform peer requests the Corrective Action Ontology to get the appropriate action.

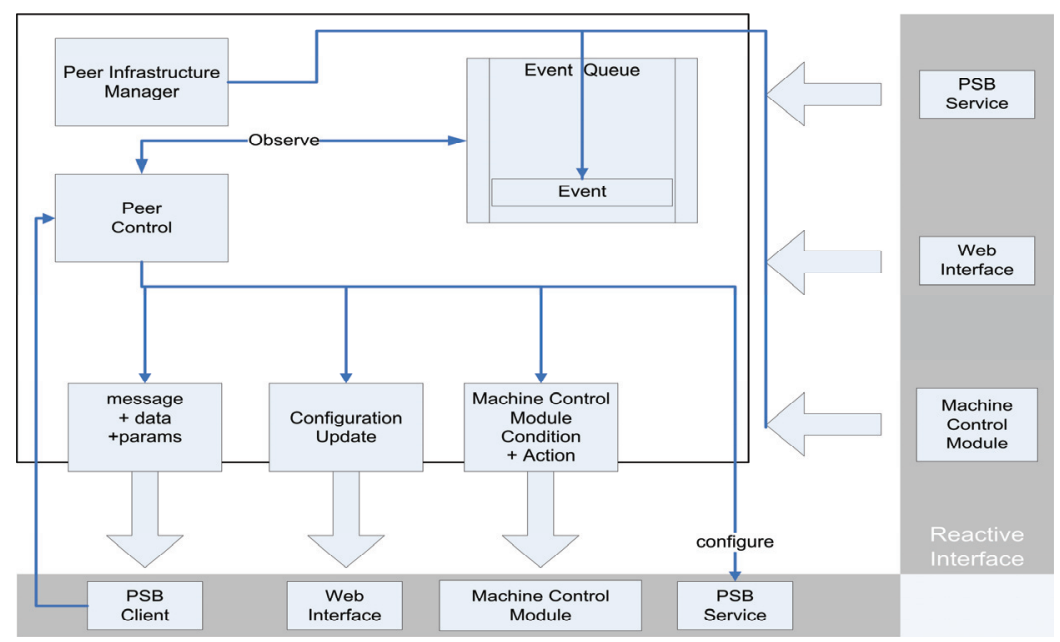

Fig. 3. Platform Peer Architecture (First Prototype)

Modules implementing the Reactive Interface are "reacting" directly to real-time requirements. In case other parts of the platform peer are concerned of such a "reaction" a peer-event is raised to trigger a more complex action. Reactions cannot become influenced directly by peer control but conditions referring to certain actions can get removed and added. This is especially important for the machine modules where event triggered actions are far too slow and not reliable.

\section{SCCP Production Service Bus (PSB)}

The SCCP communication protocol is defined in the Production Service Bus (PSB) as illustrated in figure 5. The PSB provides a service infrastructure to guarantee an easy and reliable transmission of semantic referenced data. A PSB consists of two 
4 T. Schlegel, A. Srinivasan, M. Foursa, M. Bogen, R. Narayanan, D. d'Angelo,

G. Haidegger, I. Mezgar, J. Canou, D. Sallé, F. Meo, J. Agirre Ibarbia, A. Herrmann Praturlon

layers: Peer Infrastructure Services allow implementing a decentralized peer index and contain functions that help to keep the network consistent and connected.

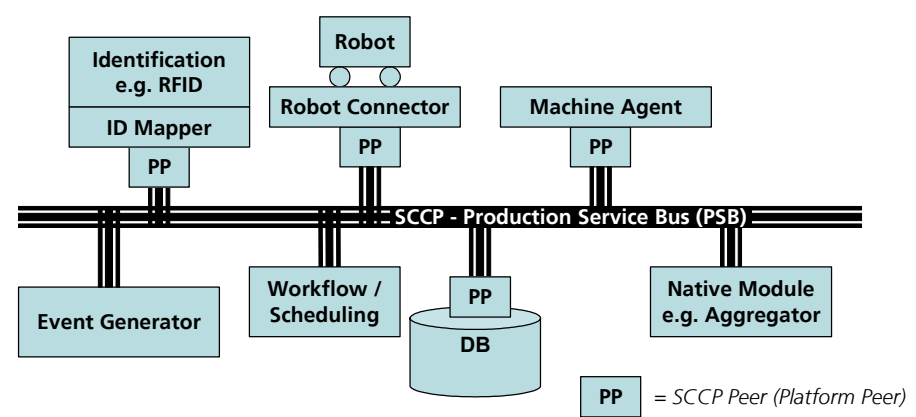

Fig. 4. PSB Bus System

The PSB describes processes for typical network situations (register, Index Node drop out, network split ...) that have to be implemented by all participating peers. The second PSB layer describes services allowing SCCP event based data transmission. On the basis of these services every peer is able to provide events to observe and can send data according to events occurred. Additionally proactive data transmission is possible as well.

\section{Ubiquitous Augmented User Interface}

To test the advanced user interfaces and demonstrate the concepts developed in the INT-MANUS project, a test bed to emulate industrial processes is needed. This section describes the design and implementation of such a test bed.

Our test bed is a model factory with machines and a robot used for the transportation of materials. This model factory makes "beams" using "cubes". The four machines are the Cube Dispenser (CD), Beam Maker (BM), Quality Controller (QC) and the Delivery Bay (DB), and the Khepera 2 robot (R) [5]. A typical working scenario of the test bed is to manufacture a 3 cube beam (a bar out of 3 cubes). The Cube Dispenser ejects a cube and the robot carries it to the cube inlet of the Beam Maker, which takes in the cube, turns it on its side, and pushes it into the beam chamber. This task is repeated 3 times and then the Beam Maker pushes out the finished beam to its outlet. The Robot carries it to the Quality Controller, where the beam is taken up a ramp and dropped from there. A beam that comes out in one piece is declared good and is taken to the delivery bay by the Robot.

\section{Structure of the First Prototype Software}

The software structure of the complete prototype is shown in Fig.5. The test bed described in the previous paragraph is shown on the Hardware layer. It is controlled by a Test bed Control System (TCS) software written in C++, which is presented in the Production processes control layer. The software communicates with the test bed embedded system via TCP/IP, with the robot via a serial link or Bluetooth, and with 


\section{INT-MANUS: Interactive Production Control}

in a Distributed Environment 5

the Mobile Control System (MCS) and tracking systems via GSoap [7]. In addition, this software can read test bed programs from an xml file, that describe an entire scenario, and execute instructions for machines one by one. Thus, the program xml file forms a script-like set of instructions for the entire test bed.

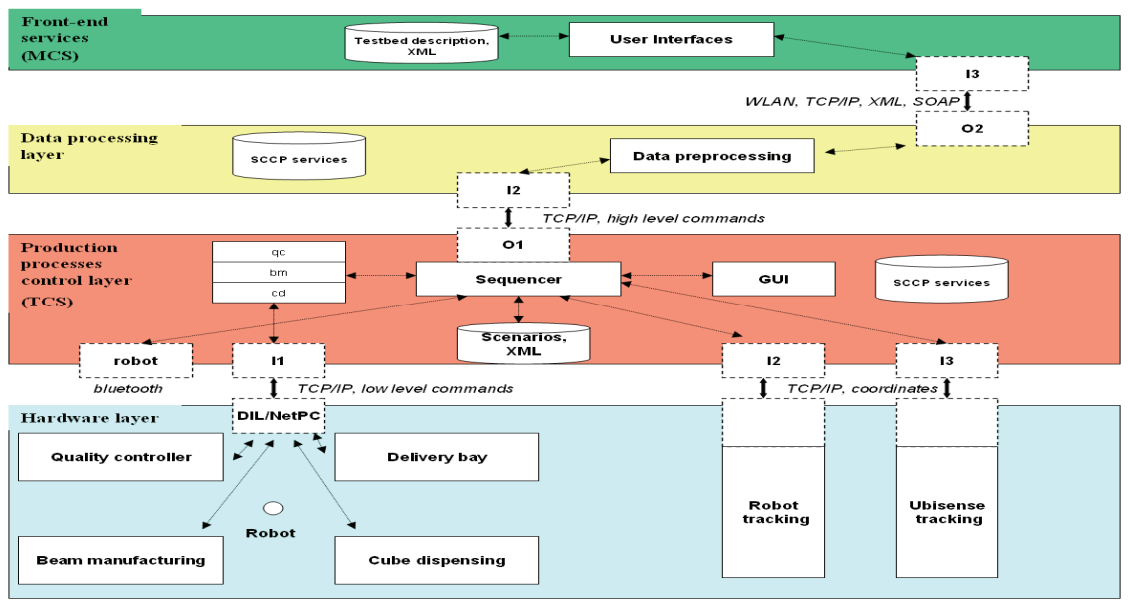

Fig. 5. Structure of the Software

The Mobile Control System (MCS) is shown on the Frond-end layer. It is running on a handheld computer SONY VAIO [8] and acts as a graphical interface for a user to get information about the industrial environment as well as for the interaction with the environment (see next paragraph). The communication between the TCS and the MCS is organized via a network abstraction layer. Due to the usage of a universal data format, the efforts for integrating with a new industrial environment are minimal, because only changes to the data processing modules must be made, while the core modules will stay the same. The TCS is responsible for the registration management of Mobile Control Systems.

\section{Virtual Reality interface}

Current industrial Virtual Reality (VR) systems [10], [11] are dedicated mostly to pure visualization or simple modeling and not connected directly to any production process. Semantic information exceeding the rather basic and numerical information needed, e.g. for FEM (finite elements method), are usually neither present nor linked to the system. This leaves a gap between the construction models edited, changed, and visualized, and the production environment data that is only reached with post processing (i.e. generation/transformation to machine specific NC code).

The INT-MANUS Virtual Reality interface, which can work closer with industrial environment, should allow flexible customization of the product in Virtual Reality directly connected to the industrial environment via the SCC platform services described above. The system receives a list of parts, which can be produced by all 
6 T. Schlegel, A. Srinivasan, M. Foursa, M. Bogen, R. Narayanan, D. d'Angelo,

G. Haidegger, I. Mezgar, J. Canou, D. Sallé, F. Meo, J. Agirre Ibarbia, A. Herrmann Praturlon

production machines as well as description of possible variants of the parts and limitations for their production. An operator can customize the product taking into account the above mentioned information.

\section{Integration of Machines and manufacturing equipment in the Interactive Production Control}

Nowadays, the Machines and manufacturing equipment are controlled by different type of control systems. In the case of machine-tools they have Open Numerical Controls, controls that offer several interfaces allowing access to the managed internal data. Other manufacturing equipments are managed by different kind of controls but in general they also offer interfaces allowing access to the managed internal data. These interfaces make it possible the integration with higher levels of the automation pyramid.

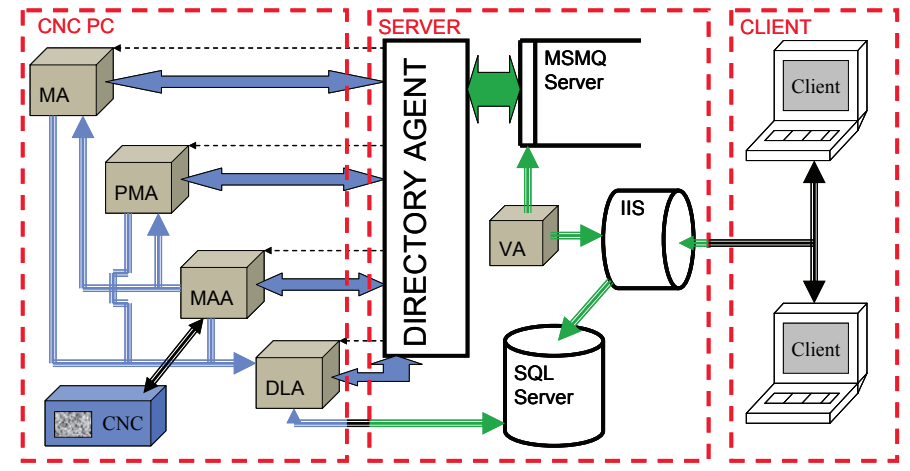

Fig. 6. SMART - Distributed scenario

In order to provide richer functionalities we use a framework based on Industrial Agents (SMART framework) that is especially devoted to a manufacturing environment. In SMART, the agents are considered as intelligent entities that can collaborate trough communication and update mechanisms. Microsoft .NET and webbased interfaces were used as the base technology. This framework offers functionality for data collection, monitoring and data logging. The SMART distributed scenario is shown in the figure.

\section{RFID}

Interactive processes are modeled using a semantic description that is directly related to the production context via instances. The identification is achieved through technologies such as RFID (Radio Frequency Identification) and optical recognition. Semantic Message communication is the key feature used in the decentralized communication process. Semantic annotation and content-based routing will enable loose coupling between components and flexible extensibility even at run-time. By 
INT-MANUS: Interactive Production Control in a Distributed Environment 7

semantic annotation, full compatibility can be maintained throughout different protocol versions. Furthermore, semantically encoded performance indicators or maintenance data can be routed along the same communication infrastructure. The SCCP communication protocol is defined in the Production Service Bus (PSB), which provides a service infrastructure to guarantee an easy and reliable transmission of semantic referenced data. The PSB consists of two layers. The Peer Infrastructure Services allow implementing a decentralized peer index and contain functions that help to keep the network consistent and connected. The second PSB layer describes services allowing SCCP event based data transmission.

\section{Maintenance Concepts}

Predictive Maintenance concepts are transferred from definition-oriented to a context-based approach supported by an adaptive system. The objective of conditionbased predictive maintenance applied to machine tools is the "measurement" of the slow degradation taking place in mechanical components in advance of the consequent failures and the evaluation of the residual life in order to better manage the total Life Cycle Cost.

The architecture selected for this achievement is shown in the following figure:

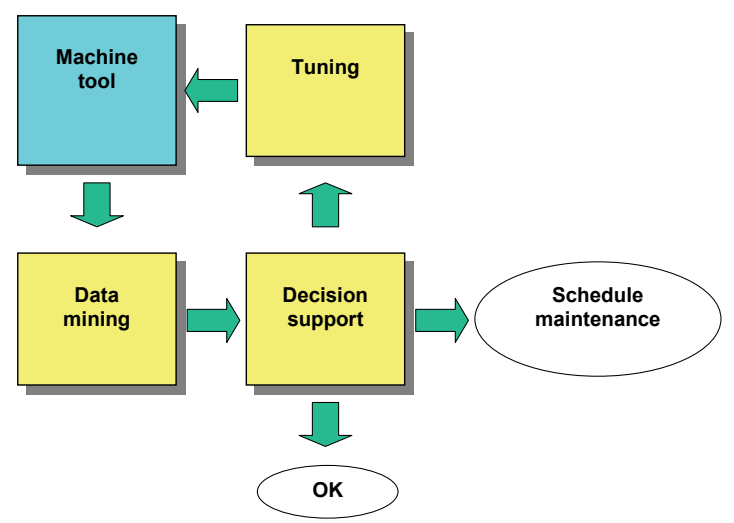

Fig. 7. Maintenance Architecture

Sensors collect data from the Machine Tool; the Data Mining software module is organized in form of a data processing pipeline and can be split in three main phases, comprising data collection from sensors with the suitable frequency, data filtering and extraction of some indices indicating the state of "health" of the mechanical parts (meaning the status of wearing out of components) like vibration critical frequency, damping factor, backlash (error at the inversion of the direction of movement), maximum and average value of current, ripple of current.

The Data Mining software provides indicators to a Decision Support module that selects the possible action, choosing among the following:

- Do nothing because the system is working very close to an optimal situation; 
8 T. Schlegel, A. Srinivasan, M. Foursa, M. Bogen, R. Narayanan, D. d'Angelo,

G. Haidegger, I. Mezgar, J. Canou, D. Sallé, F. Meo, J. Agirre Ibarbia, A. Herrmann Praturlon

- Self-tune the system, whenever possible, in order to achieve a different trade-off between accuracy and time; in fact, for instance in the production of moulds and dies, the tuning of a machine tool is usually based on the selection of a suitable compromise: if the working operation is faster, it is normally less accurate, and vice versa. The optimal compromise depends on the kind of operation; for instance during a roughing operation, accuracy is not at all required, therefore the tuning should optimize the execution time (and cost, as a consequence).

- After a certain threshold, it is not efficient anymore to slow down the machine, and an intervention for the substitution of a mechanical part is needed. The resulting reorganisation of the plant schedule should be automatically performed. The production plant supervisor should be notified about this decision and the possible reason and mechanical parts to be substituted.

In a following phase, several machine tools (some of them of the same kind, some of them of different kind) are connected to a Remote Knowledge Repository where all data are stored. Stored data are relative to:

- Previous faults;

- Results of previous evaluations of residual life;

- Results of previous tuning operations;

- Results of previous rescheduling operations.

The Knowledge Repository has to be able to transform information into knowledge, and learn by the correlation of data. For instance, applying this philosophy to diagnosis, the system will be able to correlate machine failures with factors like the climate or with the use of the machine.

\section{Robots}

The Robot developed by ROBOSOFT for the INT-MANUS project is based on a RobuLAB80 indoor mobile platform which was designed to carry high payload. The figure below shows the RobuLab80 mobile platform improved for the INT-MANUS project.

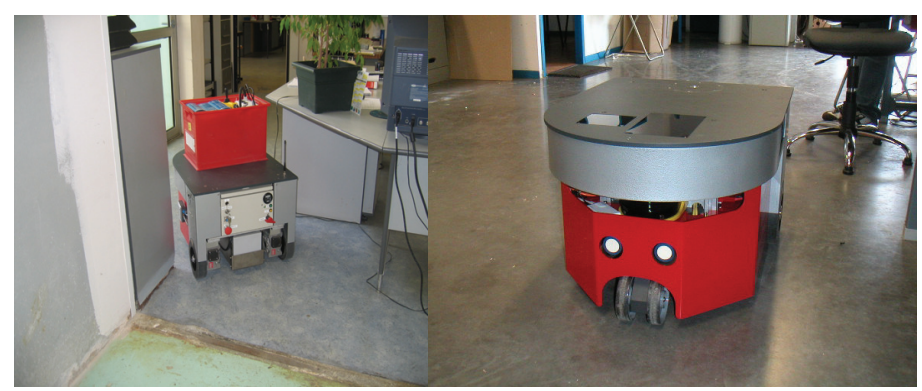

Fig. 8. Illustration of the robot mobile platform robuLAB80 
INT-MANUS: Interactive Production Control

in a Distributed Environment 9

\section{Robot software control architecture}

The robot software control architecture does not only address the capabilities of the robot to assume its own displacement. The robot displacement capabilities are a part of the global control architecture which allows also communication between SCCP, supervisor (fleet manager) and robot.

The communication between the robot side (robot and supervisor) and SCCP is made using the .Net technology from Microsoft. Running on the .Net environment ROBOSOFT has chosen the Microsoft Robotic Studio [12] product to develop its robotics control software called RobuBOX ${ }^{\mathrm{TM}}$ [13]. The basic principle is to divide a robotic application into communicating services [14], which can run on any CPU of the control system.

The control architecture of the INT-MANUS robot allows advanced maintenance by considering the failure detection of robot components (hardware and software).

\section{Fleet Supervisor}

The interface between the SCCP and the robot is made through a supervisor with fleet management capabilities. It allows to send a mission to the robot but also to monitor the state of the fleet of robots working in the factory. This fleet manager allows also the optimization of the route attached to a mission.

To allow a complete flexible and reconfigurable navigation of the robot, a set of software agents will be developed to complete this fleet manager.

These software agents will concern the detection of problematic situations and the simulation of rerouting and reassignments of the robot mission in case of failure on a production machine.

The detection of problematic situations encountered by the fleet of mobile robots while carrying out their assigned tasks, such as traffic problems, unexpected obstacles or inaccessible targets will allow reconfigurable navigation.

\section{Evaluation in the industrial environment at CRF}

For the industrial environment, the project brings high innovation in the field of customized production, allowing to achieving a complete flexibility in terms of variety of parts produced, with fast reprogramming and reconfigurability. In the case of automotive production, parts may be quickly customized, modifying the geometries and reprogramming in a smart way the whole process chain, thanks to a VR representation of the impact on the whole production flow of the plant. Therefore, a system allowing a quick (virtual) evaluation of the feasibility of a new product is needed, having access to a knowledge database in which drawings, tool paths, materials and processes are stored. After a positive outcome of the evaluation stage, the process and material flow for the new product is generated, with all the details on process time and machining scheduling inside the production plant. Finally, production can start. As it is important to test the integration of the different components, the whole prototype will be setup in order to simulate the production of an automotive component, such as an engine part, which requires an optimised 
10 T. Schlegel, A. Srinivasan, M. Foursa, M. Bogen, R. Narayanan, D. d'Angelo,

G. Haidegger, I. Mezgar, J. Canou, D. Sallé, F. Meo, J. Agirre Ibarbia, A. Herrmann Praturlon

planning of the production. In fact, in plants where high series are produced (many thousands/year), like in the automotive environment, no delays are allowed due to failures of machines and tools, because it would mean loss of production and delivery delays, with high costs for penalties to be paid by suppliers, if they are not able to provide the products on time. It follows that quick failure detection and an accurate maintenance program are of fundamental importance for the industrial overall performance. The Advanced Machining Laboratory is used to set up the demonstrator, connecting the machine tools and having a first assessment of the customisation possibilities of the parts and of the production process. The system can rely on 3D cad models of the components, which can be used and varied by an user. If the modifications fulfil the machine capabilities, the parts will be produced accordingly. The tracking system will keep an eye on every movement in the shop floor and allow optimising the production flow.

\section{Summary and Conclusion}

INT-MANUS provides a decentralized peer2peer platform for production control, integrating advanced maintenance, robot system for transport, RFID, access to numeric controls and AR / VR technology for enhanced user experience. The project will develop further two prototypes which will focus on the semantic technologies and messaging as well as advanced recognition and interaction technologies supported by a knowledge base.

INT-MANUS Project is funded by the European Commission and has emerged out of the European Network of Excellence I*PROMS, supported by its virtual Institute VIMATION.eu.

\section{References}

[1] INT-MANUS project page: www.int-manus.org

[2] I*PROMS Network of Excellence: http://www.iproms.org/

[3] World Wide Web consortium: www.w3.org

[4] robulAB80: www.robosoft.fr

[5] KHEPERA robot: www.k-team.com

[6] Solidworks: www.solidworks.com/

[7] GSOAP http://www.cs.fsu.edu/ engelen/soap.html

[8] Sony Vaio PC: http://vaio-online.sony.com/prod_info/vgn-u8g/specifications.html

[9] W3C soap: http://www.w3.org/TR/soap/

[10] VIRTOOLS http://www.virtools.com/

[11] RTT "Deltagen" http://www.realtime-technology.com/

[12] Microsoft Robotic Studio website: "http://msdn.microsft.com/robotics".

[13] White Paper robuBOXTM on Robosoft website: "http://www.robosoft.fr".

[14] DSSP specification from Microsoft website: http://download.microsoft.com/download/3/8/2/382EAA0D-576E-4F2D-803A22255A0C7251/DSSP.pdf 